\title{
MULTILEVEL SEMANTIC MODELLING OF URBAN BUILDING SPACE BASED ON THE GEOMETRIC CHARACTERISTICS IN 3D ENVIRONMENT
}

\author{
Xuan Sun \\ Zhou Enlai School of Government, Nankai University, China - sunxuan@nankai.edu.cn
}

Commission IV, WG IV/1

KEY WORDS: Semantic Modelling, Building Space, 3D Building Model, Smart City, Urban Management

\begin{abstract}
:
Data model is the basis of all the functions of geographic information system. As the land use structure has become more and more complicated in cities, the traditional geometric model are not able to satisfy the increasing demands of precise urban form recognition and space management. Against the shortcomings, we propose to construct a multilevel semantic model for better description of the spatial composition of each building and the relationships among different buildings. Based on the 3D surface models constructed with photogrammetry and remote sensing methods, the semantic model is generated to depict the urban building space hierarchically, from stories, buildings, subareas to the entire city zone. On the one hand, to figure out the stories of each building, the geometric 3D model is segmented vertically with reference to the compositional structures and spatial distributions of the functional features on the surfaces. On the other hand, to determine the subareas of the city, the buildings are grouped into meaningful clusters according to their geometric shape characteristics. Experiments were conducted on a small district with both commercial and residential buildings, and the effectiveness of the proposed approach and usage of the semantic model were demonstrated.
\end{abstract}

\section{INTRODUCTION}

With the advances of IoT (Internet of Things), cloud computing, and big data technologies, Smart City construction has become one of the major municipal projects of cities. As the abstract representation of the real world in computers, the virtual geographic environment is the most important information infrastructure of Smart City. Whereas, the traditional geometric models, which mainly serve for visualization purpose in various GIS (Geographic information systems) applications, actually do not work well in the complicated process of urban planning and management. To effectively analyze and use the land, housing and population information in cities, it is necessary to bring in the semantics and construct models that conform to the human's recognition habits and knowledge.

As the main components of urban geographic environment, buildings are closely associated with the daily life of citizens. No matter for work, entertainment, or rest, most of our activities actually happen within and between buildings. The accurate description of the indoor and outdoor space of buildings could provide a good foundation for deepening exploration of the spatiotemporal data's value and smart applications. However, due to the multiplex and comprehensive developments of cities, there are not only complicated land use structures in the horizontal direction but also diversified space utilization modes in the vertical direction. For semantic modelling of urban building space, we need to make both spatial subdivision and synthesis of the geometric building models to fit in with the demands of precise explanations at different scales.

So far, there're two kinds of semantic building models commonly used in the fields of CAD (Computer Aided Design) and Geoinformatics. The first one is BIM (Building Information Model), which is a comprehensive model covering all the design information throughout the whole life circle of architecture engineering (Cerovsek 2011, Isikdag et al. 2013).
The second is CityGML (Kolbe et al. 2005, 2009, Gröger \& Plümer 2012), which is an all-embracing data exchange model defined by OGC (Open GIS Consortium). Although it is convenient to obtain the detailed semantic information of each single building from BIM (Benner et al. 2005, Isikdag \& Zlatanova 2009), there are great difficulties to put the semantic models into practical use due to the incomplete design records of architectures and the privacy protection of construction firms. Compared with BIM, CityGML is much more widely used in the field of GIS, but the construction of the semantic model always involves a great deal of manual operation (Kelly \& Wonka 2011, Krecklau \& Kobbelt 2012). Even if some semantic information can be acquired from crowd source applications on the web (Goetz \& Zipf 2012, 2013), it is still a great challenge to generate multi-scale representations of the building space in a specified urban area.

Against the shortcomings, we propose a feasible scheme to construct the semantic model for multilevel representations of the urban building space based on the geometric characteristics in $3 \mathrm{D}$ environment. From the geometric model construction to the semantic description, the urban building space is depicted hierarchically from stories, buildings, subareas to the entire city zone. The remaining parts of this paper are organized as follows. In Section 2, the process of geometric model construction is briefly described. The methodology of semantic building space description is elaborated in details in Section 3. The experimental results of typical models are presented and discussed in Section 4. Finally, Section 5 concludes this paper.

\section{GEOMETRIC MODEL CONSTRUCTION}

Air-borne laser scanning (ALS) and terrestrial laser scanning (TLS) devices are employed to collect high-resolution point clouds of the buildings from top and side views. According to the correspondences of the outlines (Yang, et al., 2015), the 
multi-source datasets are registered to provide representations of the buildings with complete facades and roofs (see Fig. 1a).

Based on the point clouds, a synthesized approach is employed to make geometric models of the buildings with detailed features on the surfaces (see Fig.1b). On one aspect, with reference to the locally fitted plane primitives on roofs and global regularities of shapes, $2.5 \mathrm{D}$ models are automatically constructed for general representation of the buildings (Neumann \& Zhou, 2012). On another aspect, for meticulous description of the façades, smart boxes are adopted to semiautomatically model the windows and balconies of the buildings with a balance between fitting errors and mutual similarities of components (Nan et al. 2010)

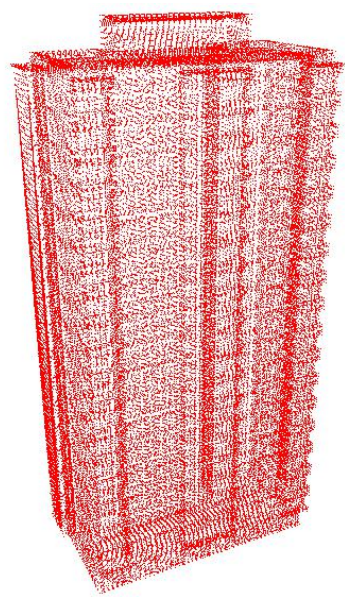

(a)

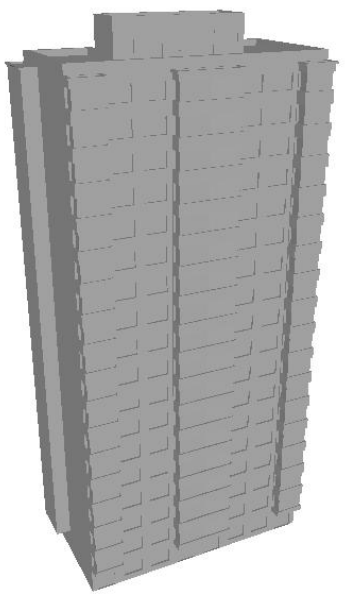

(b)
Figure 1. Geometric building model construction. (a) The registered point clouds of the building model. (b) The constructed 3D surface building model.

\section{SEMANTIC BUILDING SPACE DESCRIPTION}

Taking single building as the basic unit of housing data storage and analysis, it is hard to know exactly the space utilization of modern cities (Shojaei et al., 2013). In the micro level, the subdivisions of floor space can reflect the compositions of buildings in the vertical direction. In the macro level, the building groups and relationships of them show the urban morphology in the horizontal direction.

\subsection{Storey recognition of the building model}

Through analysis of the compositional structures of geometric shapes and the construction structures of surface features, the inner space of building models are vertically delaminated.

As the floors of buildings are invisible from outside, based on the $3 \mathrm{D}$ surface models, it is hard to extract the semantic information of floor space from the geometric models automatically with traditional methods. Nevertheless, with reference to the clues of building structures, it is possible to decompose the models into spatial units at different scales according to the basic rules of architectures. In our work, we study the overall shapes of building models firstly, to separate the them into the basic compositional parts (see Fig 2a); and then we examine the connection relationships among neighboring facets of the models, to extract all the functional features on the surfaces (see Fig 2b); finally, the spatial arrangements of features on the surface of each components are analyzed, to comprehensively deduce the floor delamination manners of building models (see Fig 2c).

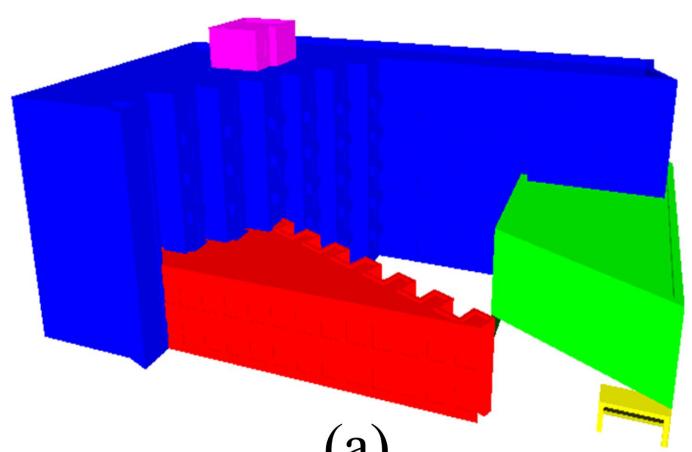

(a)
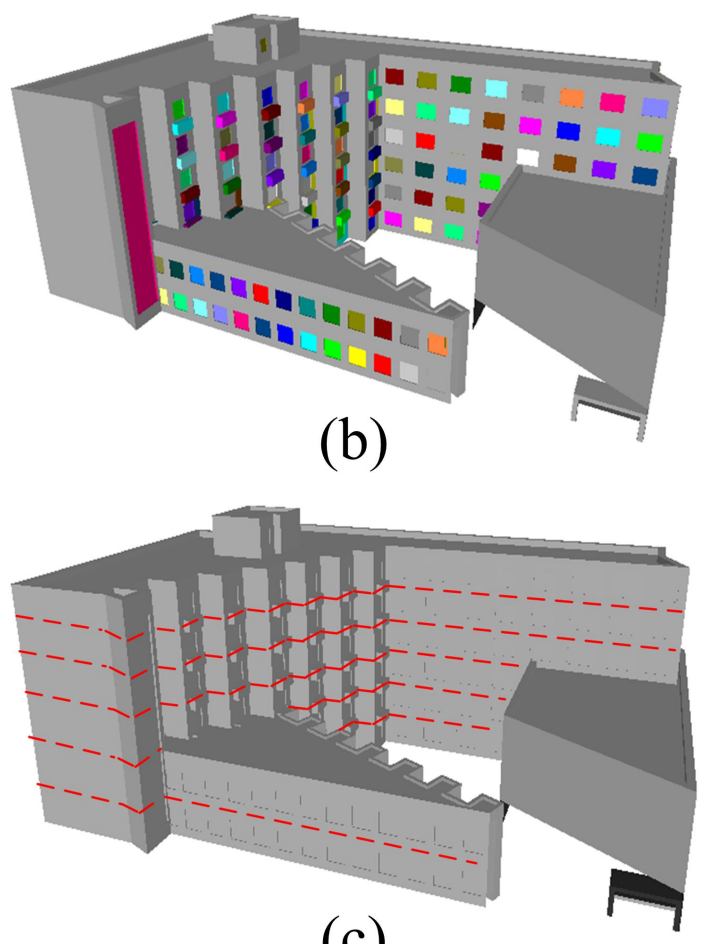

(c)

Figure 2. Storey recognition of the building model. (a) Compositional structure recognition. (b) Surface feature extraction. (c) Floor delamination on the surfaces.

Voxel analysis can not only describe the spatial occupations of models in geometry, but also reduce the influence of detailed features on the overall shapes. Thus, we proposed a method based on volumetric analysis to study the compositional structures of building models (Sun et al., 2018). Fist, the building model is voxelized, and we construct layered distance maps in the voxel space to record the shape characteristics of the model at different parts. Second, with reference to the distribution patterns of distance maps at different levels, the mid-voxels (the voxels at the middle of local parts of the building model in every level of voxels) are extracted and clustered to identify all the structural parts. Third, the other voxels are classified into different mid-voxel clusters to obtain the spatial occupations of the structural parts in the voxel space. Projecting the results back to the surface models, we can obtain the accurate representation of each part. 
Considering the complicated structures on the surfaces, geometric analysis is adopted to extract the surface features of building models ( $\mathrm{Li}$ et al., 2013). With reference to the topological relationships among different components, we divide the detailed structures of building models into three categories: the embedded structures, the composite structures, and the connecting structures. According to the convex/concave properties of every pair of neighboring faces, a topological connection graph is constructed for an abstract representation of the surface of the model. With specific rules of graph searching, the three categories of surface features are able to be effectively extracted and recognized.

\subsection{Subarea determination among building models}

The structures of building models can reflect the functionalities of the corresponding architectures in some degree, e.g. the commercial buildings and the attraction landmarks tend to have complicated structures, and the residential buildings are always with simple shape and the function features closely related with the daily lives of people. Taking the structure properties of building models as a special type of semantic information, together with the spatial relationships, the urban space is able to be segmented into meaningful blocks, within which all the building models exhibit similar structure characteristics (see Fig3).

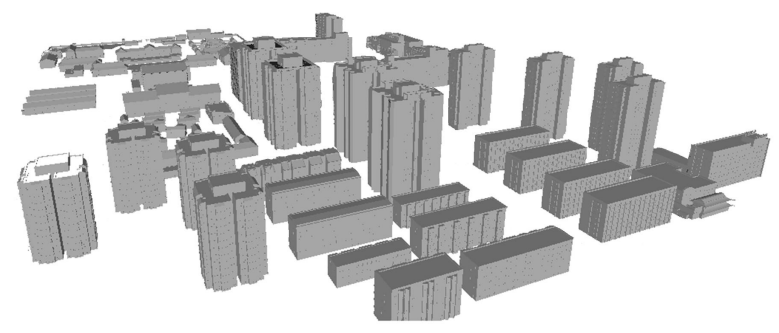

(a)

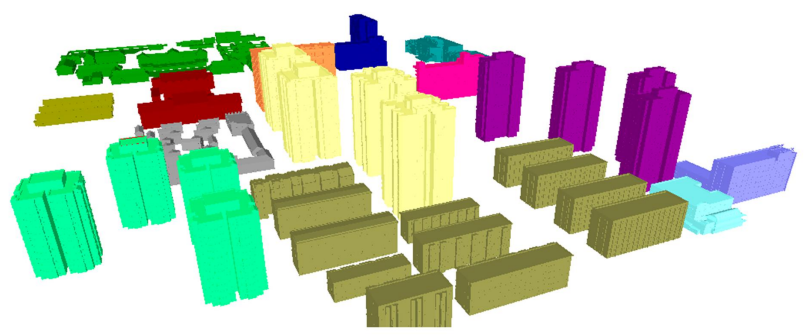

(b)

Figure 3. Subarea determination among building models. (a) The geometric building models in certain city zone. (b) The building models grouped according to their structure characteristics.

First, the distance of the nearest points of the footprints is calculated for judgment of the neighboring relationship of every two building models. If the distance is less than the threshold, e.g. 50 meters, the two building models are regarded as neighboring to each other; otherwise, they are not. Second, based on the preliminary judgments of the spatial linkages, the semantic correlation of building models is further discussed to determine the subdivision scheme of land space.

By analyzing the structural characteristics of various types of building models, a quantitative classifier is designed for semantic grouping. Specifically, the volume of the biggest compositional structural part, the number of compositional structural parts, the number of embedded structures, the number of composite structures, and the number of connecting structures are mainly considered to describe the shape and function features of building models. Assigning different weights to the five parameters, the structure variation of any two building model can be calculated with following formula:

$\operatorname{Sc}\left(b_{i}, b_{j}\right)=\sum_{k}^{5} \omega_{k}\left|P_{k}\left(b_{i}\right)-P_{k}\left(b_{j}\right)\right|$

$b_{i}$ and $b_{j}$ are two different building models, Sc is the variation score of the two building models, $P_{k}$ stands for the $k$ parameter of any of the models, and $\omega_{k}$ stands for the weight for the $k$ parameter. With reference to the variation score, the difference of building models in structures can be measured.

\subsection{Multi-level organization and representation of building space}

Based on the semantic relationships of the floor space, buildings, and building groups, the 3D building models are reconstructed and synthesized. By establishing a unified data structure, the multilevel urban building space is hierarchically organized and expressed. The key of multilevel representation of building space is the consistency of geometry and semantics.

On the one hand, according to the level-by-level description result of the floor space, the $3 \mathrm{D}$ building models are able to be vertically segmented on the surfaces. Considering the topological relationship between adjacent floors and between the floors and the buildings, the triangular patches across multiple floors are subdivided and reconstructed along dividing line of the floors on the surfaces of the original models. When each geometric element of the outer surface of the model is assigned to the specific floor space to which it belongs, the consistent expression of the single building and the interior floor space of the building is achieved.

On the other hand, to obtain the abstract representation of building group space, the synthesis method is used to merge and simplify the multiple models in the same building group. In the horizontal direction, with reference to the spatial relationships among the building models, the ground contours of the 3D model are merged. In the vertical direction, the average height of the building group is determined according to the floor height and the plot ratio of the corresponding block area.

With reference to the ways that buildings are grouped and floor delamination modes, the semantic objects at different levels are organized together to construct a hierarchical framework. Based on the hierarchical framework, the semantic information and geometries are all stored and managed in a unified manner.

\section{EXPERIMENTS AND APPLICATIONS}

To testify the proposed approach of multilevel semantic modelling of urban building space, a small urban area in the real world is chosen for experiments. In the trial area, there are more than 500 architectures, including residential buildings, commercial buildings and some civic buildings.

\subsection{Geometric modelling of the buildings}

The geometric data of the buildings was collected with Riegl LMS-Q160 ALS device and Riegl VZ400 TLS device, and the resolutions of the point clouds obtained were respectively 8 
points and 12 points per square meters. After registration, more than 600 million points were merged together to provide detailed representation of the trial area.

With the high-resolution point clouds, 3D building models were generated through the semi-automatic modelling method (see Section 2). Composed of 1.16 million triangular facets, all the function features of buildings were well recorded on the surfaces of the models (see Fig4)

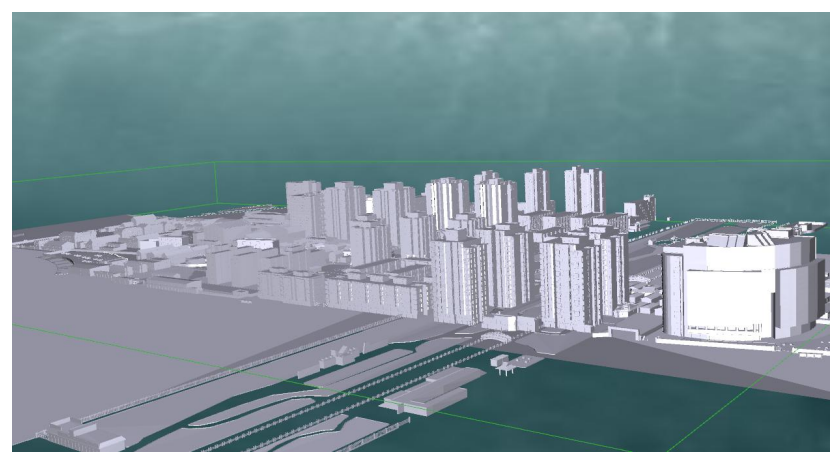

Figure 4. geometric modelling of the buildings.

\subsection{Multilevel description of the building space}

Based on the 3D geometric building models, a multilevel semantic model was constructed (see Fig.5).

In the macro perspective, the buildings were clustered into 14 groups. While some groups had more than 10 building models, there were also groups that only composed of one building model. With the diversified building groups, the function zones and landmarks in the trial area were clearly depicted.

In the micro perspective, the building models were segmented into a number of floors at different levels. The models with complicated structures in the vertical direction were composed of floor spaces with various shapes. Nevertheless, the models with simple structures in the vertical direction were always decomposed into floor spaces with consistent shape.

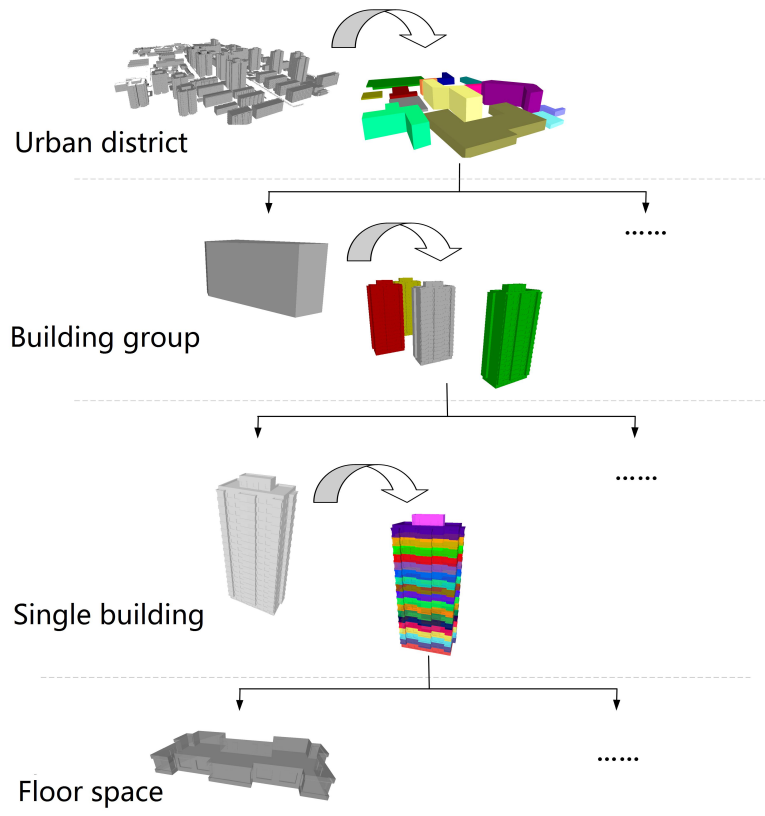

Figure 5. Multilevel description of the building space.

\section{CONCLUSION}

In this paper, we propose to construct a multilevel semantic model for unified description and management of urban building space at different scales. The obtained semantic model not only can clearly depict the layered floor space of the building in the vertical direction, to satisfy the demands of fine management of population and real estate information, but also can illustrate the building group space in the horizontal direction, to meet the needs of urban spatial form recognition. It expands the application scope of 3D geographic information system in the field of urban planning and management, and provides technical support for the construction of smart cities.

Our research is just an initial step of semantic modelling. For precise and effective description of urban space, much more works still need to be done in the future. On one aspect, how to make detailed description of the apartments in each floor of the building based on geometric models or other sources of data is still under challenge. On another aspect, the building groups can actually be generated with different scales and from different perspectives, yet it is a complicated problem to choose proper control parameters and to set appropriate thresholds for accurate expression of semantics.

\section{ACKNOWLEDGEMENTS}

This study is jointly supported by the National Natural Science Foundation of China (Grant No. 41501427), the Fundamental Research Funds for the Central Universities (Grant No. NKZXB1483), the Project of Asia Research Center in Nankai University (Grant No. AS1522).

\section{REFERENCES}

Benner, J., Geiger, A., Leinemann K., 2005. Flexible generation of semantic 3D building models. In: Proceedings of the 1 st international workshop on next generation $3 D$ city models, Bonn, pp. 17-22.

Cerovsek, T., 2011. A review and outlook for a 'Building Information Model' (BIM): a multi-standpoint framework for technological development. Advanced Engineering Informatics, 25(2), pp. 224-244.

Goetz, M and Zipf, A., 2012. Towards defining a framework for the automatic derivation of 3D CityGML models from volunteered geographic information. International Journal of 3 D Information Modeling, 1(2), pp. 1-16.

Goetz, M., 2013. Towards generating highly detailed 3D CityGML models from OpenStreetMap. International Journal of Geographical Information Science, 27(5), pp. 845-865.

Gröger, G. and Plümer L., 2012. CityGML-Interoperable semantic 3D city models. ISPRS Journal of Photogrammetry and Remote Sensing, 71, pp. 12-33.

Isikdag, U. and Zlatanova, S., 2009. Towards defining a framework for automatic generation of buildings in CityGML using building Information Models. In: 3D Geoinformation and Sciences, Springer, Berlin, Heidelberg, pp. 79-96.

Isikdag, U., Zlatanova, S., Underwood, J., 2013. A BIMOriented Model for supporting indoor navigation requirements. Computers, Environment and Urban Systems, 41, pp. 112-123. 
Kelly, T. and Wonka, P., 2011. Interactive architectural modeling with procedural extrusions. ACM Transactions on Graphics (TOG), 30(2), pp. 1-15.

Kolbe, T. H., Gröger G., Plümer L., 2005. CityGML: Interoperable access to 3D city models. In: Geo-information for Desaster Management. Springer, Berlin, Heidelberg, pp. 883899.

Kolbe T. H., 2009. Representing and exchanging 3D city models with CityGML. In: $3 D$ geo-information sciences, Springer, Berlin, Heidelberg, pp. 15-31.

Krecklau, L. and Kobbelt, L., 2012. Interactive modeling by procedural high-level primitives. Computers \& Graphics, 36(5), pp. 376-386.

Li, Q., Sun X., Yang B., Jiang S., 2013. Geometric Structure simplification of 3D building models. ISPRS Journal of Photogrametry and Remote Sensing, 84, pp. 100-113.

Nan, L., Sharf, A., Zhang H., Cohen-Or D., Chen B, 2010. SmartBoxes for Interactive Urban Reconstruction. $A C M$ Transactions on Graphics (SIGGRAPH), 29(4), pp. 1-11.

Neumann, U and Zhou, Q. Y., 2012. 2.5 D building modeling by discovering global regularities, In: Proceedings of IEEE Conference on Computer Vision and Pattern Recognition, pp. 326-333.

Shojaei, D., Kalantari, M., Bishop I., Rajabifard, A., Aien, A., 2013. Visualization requirements for $3 \mathrm{D}$ cadastral systems. Computers, Environment and Urban Systems, 41, pp. 39-54.

Sun, X., Li, Q., Yang, B., 2018. Compositional Structure Recognition of 3D Building Models Through Volumetric Analysis, IEEE Access, 6, pp. 33953-33968.

Yang, B., Zang, Y., Dong, Z., Huang, R., 2015. An Automated Method to Register Airborne and Terrestrial Laser Scanning Point Clouds, ISPRS Journal of Photogrammetry and Remote Sensing, 109, pp. 62-76. 